\title{
New results for Shannon capacity over generalized multipath fading channels with MRC diversity
}

\author{
Faissal El Bouanani ${ }^{1 *}$, Hussain Ben-Azza ${ }^{2}$ and Mostafa Belkasmi ${ }^{1}$
}

\begin{abstract}
In this article, we investigate the Shannon capacity for L-branch maximal combining ratio (MRC) over generalized multipath fading channel. We derive closed-form expressions of the maximal spectral efficiency over Rayleigh, Rician, Nakagami-m, and Weibull multipath fading channel under flat fading conditions. The results are expressed in terms of Meijer G-functions, which can be evaluated numerically using mathematical tools such as Mathematica and Maple. We show, in particular, that the more the number $L$ increases, the larger the Shannon capacity is. We deduce that four branches are sufficient in several cases to mitigate the fading effect and the channel model will approaches the one of AWGN.
\end{abstract}

Keywords: Shannon capacity, Spectral efficiency, DS-CDMA, Multipath fading channels, Meijer G-functions, Wireless communication, Rake receiver, MRC diversity

\section{Introduction}

The channel capacity is an important parameter in the design of any communication system. It provides an upper bound of maximum transmission rate in a given channel. In 1948, Shannon derived the AWGN channel capacity $[1,2]$. Recently, in wireless mobile communication system, the diversity techniques have been used to combat multipath fading and multiuser interference [3].

In last years, several articles have been published regarding the Shannon capacity of fading channels with various important diversity schemes, such as maximum ratio combining (MRC), postdetection equal gain combining (EGC), and selective combining (SC), in terms of generalized special functions. The capacity with MRC in correlated Rayleigh fading in terms of Poisson distribution and Exponential integral was obtained in $[4,5]$. In [6,7], an expression of the capacity of single branch receivers operating over Rician, Nakagami- $m$, and Weibull fading channel was obtained in term of Meijer G-function. Some statistics properties, such as the probability density function (PDF) and the cumulative distribution function, of

*Correspondence: elbouanani@ensias.ma

1 ENSIAS, Mohammed V-Souissi University, Rabat, Morocco

Full list of author information is available at the end of the article the instantaneous signal-to-noise ratio (SNR) per symbol at the output of MRC receiver in correlated Nakagami$m$ fading was derived in terms of Fox's $\mathrm{H}$ and Gamma functions $[8]$ in $[9,10]$. A statistical analysis for the capacity over Nakagami- $m$ fading with $\mathrm{MRC} / \mathrm{SC} /$ switch and stay combining (SSC) in terms of Meijer G-function was presented in [11]. In [12], the capacity expressions of correlated Nakagami- $m$ fading with sual-branch MRC, EGC, SC, and SSC were obtained in terms of Gamma function. Expressions for the capacity of generalized fading channel with MRC/EGC for MIMO/SISO systems based on moment generating function (MGF) approach was obtained in terms of Fox's $H$ and Meijer functions $[13,14]$. Recently, a novel expression for the BER of modulations and Shannon capacity over generalized-K and Nakagami$m$ fading channels in terms of Meijer G-function and its generalization [15] was investigated in [16,17].

The equivalent channel model of a multipath fading channel using a MRC Rake receiver and flat fading has been approximated by a fading channel with fading amplitude is a square root of a sum of a square amplitude of each fading $[18,19]$. The equivalent channel model in DSCDMA system with MRC-Rake receiver was investigated in [18].

\section{Springer}


In this article, we present novel closed-form and analytical expressions, in terms of Meijer G-function, for the ergodic Shannon capacity for $L$-branch MRC-Rake receiver over Rician, Rayleigh, Nakagami- $m$, and simple approximation for Weibull multipath fading channel in the non-frequency selective channels case. We generalize for $L$-branch MRC the capacity expression given for single path case $(L=1)$ in [6,7]. All the results are validated by numerical Monte Carlo simulations. The study include both DS-CDMA system and no-spreading system cases.

This article is structured as follows. In Section 'Channel model', the equivalent channel models of both DS-CDMA communication system and system without spreading using a Rake receiver is introduced. In Section 'Channel capacity', the closed-form expression of the channel capacity for multipath fading channel (case of Rayleigh, Rice, Nakagami- $m$, and Weibull) is derived. The main results are summarized and some conclusions are given in Section 'Conclusion'. For the convenience of the reader, an short appendix is added, regarding Meijer G-function.

\section{Channel model}

In this section, we present the equivalent channel model of communication systems using coherent MRC receiver in both system without spreading and Direct spread spectrum system (DS-CDMA).

\section{System with MRC diversity}

Consider MRC diversity systems in flat fading environment. Let

- $x_{i}, y_{i}, b_{i}$ be the $i$ th transmitted symbol, $i$ th combined received symbol, $i$ th zero-mean, $N_{0} / 2$-variance Gaussian noise added,

- $N_{0}$ is the noise power spectral density,

- $h_{i l}$ be the fading amplitude corresponding to the $i$ th symbol and the 1 th antenna, assumed being i.i.d.,

- $L$ be the number of diversity channels,

- $E_{s}$ be the average symbol energy.

The instantaneous combined received symbol energyto-noise at the output of the MRC is $\gamma=\frac{E_{s}}{N_{0}} \sum_{l=1}^{L} h_{i l}^{2}[19,20]$, and then the normalized fading amplitude of channel is given by:

$$
A_{i L}=\sqrt{\frac{\sum_{l=1}^{L} h_{i l}^{2}}{\sum_{l=1}^{L} \mathbb{E}\left[h_{i l}^{2}\right]},}
$$

where $\mathbb{E}[$.$] denotes the expectation operator. The multi-$ path channel model with MRC diversity can be written as

$$
y_{i}=A_{i L} x_{i}+b_{i}
$$

\section{DS-CDMA system}

Let $x_{i}^{(k)}$ and $y_{i}^{(k)}$ be, respectively, the $i$ th transmitted and received code symbol of the $k$ th user over a multipath fading channel of the uplink DS-CDMA system. In [18], it is shown (see Figure 1) that for random spreading sequences, the combination of the inner interleaver/deinterleaver pair, the quadrature spreader, the transmit/receive filter pair, the frequency up/down converter pair, the fading multipath channel, and the Rake receiver can be accurately modeled by the memoryless channel of input $x_{i}^{(k)}$ and output $y_{i}^{(k)}$ given by:

$y_{i}^{(k)}=\left[\left(g \sum_{l=1}^{L}\left|h_{i l}^{(k)}\right|^{2}\right)^{1 / 2} x_{i}^{(k)}+\sqrt{I_{0} / 2} b_{i}^{(k)}\right]\left(g \sum_{l=1}^{L}\left|h_{i l}^{(k)}\right|^{2}\right)^{1 / 2}$

where:

- $b_{i}^{(k)}$ represents the zero-mean, unit-variance Gaussian noise added to the $i$ th transmitted code symbol of the $k$ th user,

- $\left|h_{i l}^{(k)}\right|$ is the fading amplitude associated to the $k$ th user, the lth path and the ith symbol (the $h_{i l}^{(k)}$ are complex numbers). The amplitudes are assumed independent identically distributed (i.i.d.)

- $L$ is the Rake receiver branch number,

- $g$ is the spreading factor,

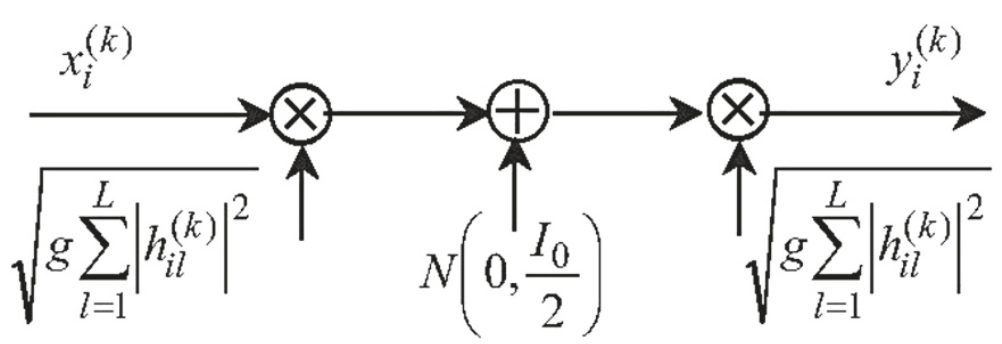

Figure 1 Equivalent channel model. 
- $I_{0}$ is the total noise power spectral density (including multiple access interference, thermal noise, and multipath fading),

For a cell with $K$ users where the channel energy of each user is normalized to $1\left(\mathbb{E}\left[\left(A_{i L}^{(k)}\right)^{2}\right]=1\right)$, the channel model (3) becomes:

$$
y_{i}^{(k)}=\left(A_{i L}^{(k)} x_{i}^{(k)}+\sqrt{\frac{I_{0}}{2}} b_{i}^{(k)}\right) A_{i L}^{(k)},
$$

where $A_{i L}^{(k)}=\sqrt{\frac{\sum_{l=1}^{L}\left|h_{i l}^{(k)}\right|^{2}}{\sum_{l=1}^{L} \mathbb{E}\left[\left|h_{i l}^{(k)}\right|^{2}\right]}}$.

Remark 1. From (4) and (1), we can see that the equivalent fading amplitude in both DS-CDMA and general case of system without spreading using MRC technique is the same. Consequently, the spectral efficiency is the same in the two cases.

For simplicity of notation, and regardless of the user and bit index, we use $I_{0}$ as the total noise power, the $A_{L}$ as the equivalent fading amplitude, and $h_{l}$ as the $l$ th path fading amplitude (that is, we drop the index $i$ and index $k$ ).

\section{Channel capacity}

The Shannon capacity of a fading channel is the average of AWGN channel capacity where the input is multiplied by the normalized fading amplitude $r$. In the case of a flat fading, $r$ is assumed to be constant over the symbol duration. Consequently, the energy by symbol becomes $E=E_{s} r^{2}$, and the average capacity of a fading channel is a function of the bandwidth $W$, the instantaneous received SNR $\gamma=\bar{\gamma} r^{2}$

$$
C=W \mathbb{E}_{R}\left[\log _{2}\left(1+\bar{\gamma} R^{2}\right)\right]=W \int_{0}^{+\infty} \log _{2}\left(1+\bar{\gamma} r^{2}\right) f(r) d r
$$

where $\bar{\gamma}=\frac{E_{s}}{T W I_{0}}$ is the average received SNR, $T$ is the symbol period, and $f(r)$ denotes the probability density function (PDF) of the fading amplitude random variable $R$.

In the following, we give the exact Shannon capacity (5) for the channel models described in the previous section in the case where all $\left|h_{i l}^{(k)}\right|$ are Rayleigh, Rician, Nakagami$m$ or Weibull distributed random variables (RVs).

\section{Rayleigh multipath fading channel}

The Rayleigh multipath fading channel is a model of a channel where the receiver can't receive a direct signal line of sight (LOS) from the source. All received signals are diffracted, reflected or diffused. In this case, the fading of $j$ th path is given by

$$
R=\sqrt{X_{j 1}^{2}+X_{j 2}^{2}}, \quad 1 \leq j \leq L,
$$

where $X_{j 1}$ (in-phase component) and $X_{j 2}$ (quadrature component) are independent normally distributed RVs with mean 0 and the same variance $\sigma^{2}$.

$$
\text { Consequently, the } \mathrm{RV} \sqrt{\sum_{j=1}^{L} \sum_{k=1}^{2}\left(X_{j k} / \sigma\right)^{2}} \text { is a central chi }
$$

distribution of $2 L$ degrees of freedom, and its $\mathrm{pdf}$ is given by

$$
f(r)=\frac{2^{1-L} r^{2 L-1} e^{-\frac{r^{2}}{2}}}{\Gamma(L)}
$$

where $\Gamma(L)=\int_{0}^{+\infty} t^{L-1} e^{-t} d t$ denotes the gamma function [8].

Thus, the PDF of the normalized fading amplitude $\frac{1}{\sqrt{2 L} \sigma} \sqrt{\sum_{j=1}^{L} \sum_{k=1}^{2} X_{j k}^{2}}$ can be expressed as

$$
f(r)=\frac{2 L^{L} r^{2 L-1} e^{-L r^{2}}}{\Gamma(L)}
$$

From (5), the equivalent channel capacity is

$$
C=W \int_{0}^{+\infty} \log _{2}\left(1+\bar{\gamma} r^{2}\right) \frac{2 L^{L} r^{2 L-1} e^{-L r^{2}}}{\Gamma(L)} d r
$$

Using the change of variables $\gamma=\bar{\gamma} r^{2}$, and the equality $\Gamma(L)=(L-1) ![8]$, the above expression can also be expressed as

$$
\frac{C}{W}=\frac{\left(\frac{L}{\bar{\gamma}}\right)^{L}}{\ln (2)(L-1) !} \int_{0}^{+\infty} \ln (1+\gamma) \gamma^{L-1} e^{-\frac{L}{\gamma} \gamma} d \gamma
$$

Let $G_{p, q}^{m, n}\left[x \mid \begin{array}{l}a_{1}, \ldots, a_{n}, \ldots, a_{p} \\ b_{1}, \ldots, b_{m}, \ldots, b_{q}\end{array}\right]$ be the Meijer Gfunction [21] where $m \leq q, n \leq p$, and $\left(a_{i}\right)_{1 \leq i \leq p},\left(b_{i}\right)_{1 \leq i \leq q}$ are two complex sequences. Using the transformation formulas (42), (43), and (46) listed in Appendix 1:

$$
e^{-\frac{\gamma L}{\bar{\gamma}}}=G_{0,1}^{1,0}\left[\frac{L}{\bar{\gamma}} \gamma \mid 0\right]
$$

and:

$$
\ln (1+\gamma)=G_{2,2}^{1,2}\left[\begin{array}{l|l}
\gamma & 1,1 \\
1,0
\end{array}\right]
$$

and (see (46) of the Appendix 1):

$$
\begin{gathered}
\int_{0}^{+\infty} G_{2,2}^{1,2}\left[\gamma \mid \begin{array}{l}
1,1 \\
1,0
\end{array}\right] G_{0,1}^{1,0}\left[\frac{L}{\bar{\gamma}} \gamma \mid 0\right] \gamma^{L-1} d \gamma \\
=G_{2,3}^{3,1}\left[\begin{array}{l|l}
\left.\frac{L}{\bar{\gamma}} \mid \begin{array}{l}
-L, 1-L \\
0,-L,-L
\end{array}\right]
\end{array}\right.
\end{gathered}
$$


we obtain the exact expression of the normalized average capacity (maximal spectral efficiency):

$$
\frac{C}{W}=\frac{\left(\frac{L}{\bar{\gamma}}\right)^{L}}{\ln (2)(L-1) !} G_{2,3}^{3,1}\left[\frac{L}{\bar{\gamma}} \mid \begin{array}{c}
-L, 1-L \\
0,-L,-L
\end{array}\right]
$$

For single-path Rayleigh fading channel $(L=1)$, the expression (13) is exactly that found by Sagias et al. [6] using the single branch receiver (SBR). This is due to the fact that the single finger Rake receiver and SBR have identical function.

The Figures 2a,b depict the effect of fingers number $L$ on the average Shannon capacity obtained analytically from (13) and via Monte Carlo simulation from (5) by generating 2500 Rayleigh-distributed random values. It is shown that as $L$ increases, the capacity increases and converges to that of the AWGN channel. These curves show again that the values $L=4$ appears to be practically sufficient to achieve the AWGN channel capacity and to eliminate the fading effect.

The Figure 3 shows the PDF of the Rayleigh multipath flat fading amplitude for $L=3,4$, and 6 . The curves show that for great values of $L$, the PDF is infinite for $r=1$ (no fading). Thus, the channel studied converge to the AWGN one.

\section{Rician multipath fading channel}

In this case of fading, the mobile receive, in addition to the other non LOS components, a direct signal from the source. The fading of the $j$ th path is a Rician distributed and can be modelized by:

$$
R_{j}=\sqrt{X_{j 1}^{2}+X_{j 2}^{2}}, \quad 1 \leq j \leq L,
$$

where $X_{j 1}$ (in-phase component) and $X_{j 2}$ (quadrature component) are independent normally distributed RV with the same variance $\sigma^{2}$ and means $s_{j} \cos \gamma_{j}$ and $s_{j} \sin \gamma_{j}$, respectively, $\left(\gamma_{j}\right.$ is a random real number and $s_{j}$ is the LOS amplitude of the $j$ th path Rician fading). Thus, the normalized $A_{L}$ is:

$$
A_{L}=\frac{\sqrt{\sum_{j=1}^{L} \sum_{k=1}^{2} X_{j k}^{2}}}{\sqrt{\sum_{j=1}^{L} s_{j}^{2}+2 L \sigma^{2}}}
$$

Let's note $\beta=\left(\sum_{j=1}^{L}\left(s_{j} / \sigma\right)^{2}+2 L\right)^{1 / 2}$. The RV $\beta A_{L}$ is a non-central chi distribution of $2 L$ degrees of freedom, and non-centrality parameter $\lambda=\left(\sum_{j=1}^{L}\left(s_{j} / \sigma\right)^{2}\right)^{1 / 2}$. Its PDF is known to be [22]

$$
f(r)=\lambda I_{L-1}(\lambda r)\left(\frac{r}{\lambda}\right)^{L} e^{-\frac{r^{2}+\lambda^{2}}{2}},
$$

where $I_{L-1}($.$) is a modified Bessel function of the first kind$ of order $L-1$ [8].

Using the Jacobian transformation method, the PDF of $A_{L}$ is given by

$$
f(r)=\beta \lambda I_{L-1}(\lambda \beta r)\left(\frac{\beta r}{\lambda}\right)^{L} e^{-\frac{(\beta r)^{2}+\lambda^{2}}{2}}
$$

The Shannon capacity for the equivalent channel (3) is then

$$
C=W \beta \lambda e^{-\frac{\lambda^{2}}{2}} \int_{0}^{+\infty} \log _{2}\left(1+\bar{\gamma} r^{2}\right) I_{L-1}(\lambda \beta r)\left(\frac{\beta r}{\lambda}\right)^{L} e^{-\frac{(\beta r)^{2}}{2}} d r
$$

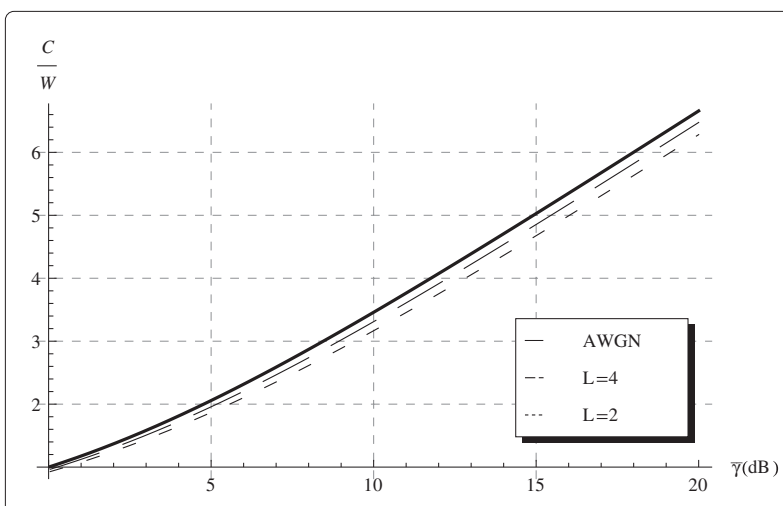

(a) Analytical expression

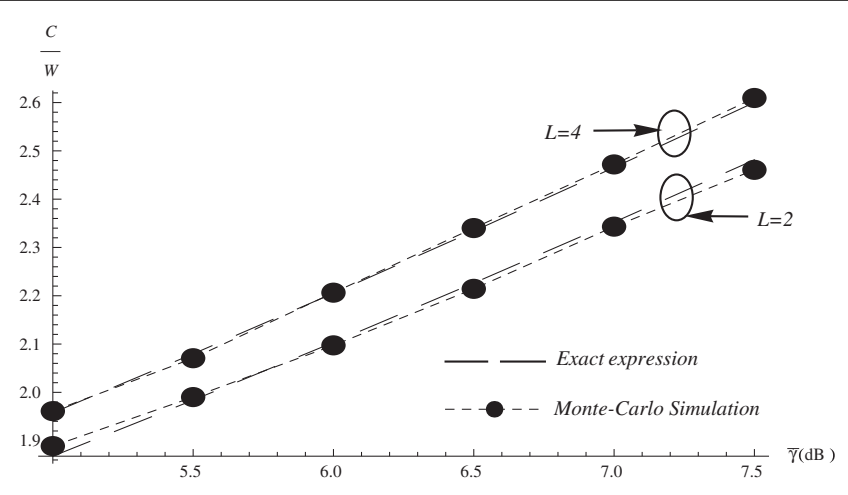

(b) Simulation and analytical expressions

Figure 2 Normalized average channel capacity versus average received SNR in Rayleigh L-path fading channel. (a) Analytical expression. (b) Simulation and analytical expressions. 


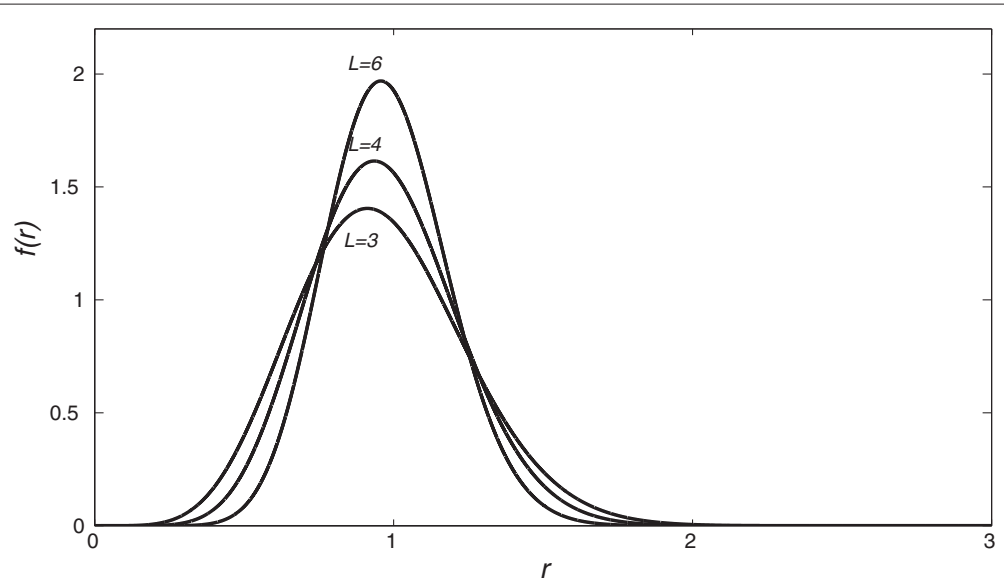

Figure 3 PDF of the Normalized fading amplitude $A_{L}$ in Rayleigh $L$-path fading channel.

Let's note $\phi=\frac{\lambda \beta}{\sqrt{\bar{\gamma}}}$. Using the change of variable $\gamma=$ $\bar{\gamma} r^{2}$, we deduce that

$\frac{C}{W}=\frac{\phi^{L+1} e^{-\frac{\lambda^{2}}{2}}}{2 \ln (2) \lambda^{2 L}} \int_{0}^{+\infty} \ln (1+\gamma) I_{L-1}(\phi \sqrt{\gamma}) e^{-\frac{\beta^{2} \gamma}{2 \ddot{\gamma}}} \gamma^{\frac{L-1}{2}} d \gamma$

Since the modified Bessel function of the first kind can be written as the infinite series ([23], BesselAiryStruveFunctions/BesselI/06/01/01/)

$$
I_{L-1}(\phi \sqrt{\gamma})=\left(\frac{\phi \sqrt{\gamma}}{2}\right)^{L-1} \sum_{j=0}^{+\infty} \frac{1}{\Gamma(j+L) j !}\left(\frac{\phi \sqrt{\gamma}}{2}\right)^{2 j}
$$

the normalized Shannon capacity can also be written as

$$
\begin{aligned}
\frac{C}{W}= & \frac{e^{-\frac{\lambda^{2}}{2}}}{\ln (2)}\left(\frac{\beta^{2}}{2 \bar{\gamma}}\right) \sum_{j=0}^{L+\infty} \frac{1}{(j+L-1) ! j !}\left(\frac{\phi}{2}\right)^{2 j} \\
& \times G_{2,3}^{3,1}\left[\frac{\beta^{2}}{2 \bar{\gamma}} \mid \begin{array}{c}
-j-L, 1-j-L \\
0,-j-L,-j-L
\end{array}\right]
\end{aligned}
$$

This formula generalizes the capacity expression founded by Sagias et al. [6] in the case of one path. It generalizes also our first result corresponding to the normalized Rayleigh multipath fading channel. Indeed, for Rayleigh fading, all LOS amplitudes $s_{j}$ equal zero $(\lambda=\phi=$ $0, \frac{\beta^{2}}{2}=L$ ), and then we find the above expression (13).

The two Figures $4 \mathrm{a}, \mathrm{b}$ show the normalized average Shannon capacity of a Rician multipath fading channel obtained analytically from (21) and via simulation from (5). The average energies associated with each path are

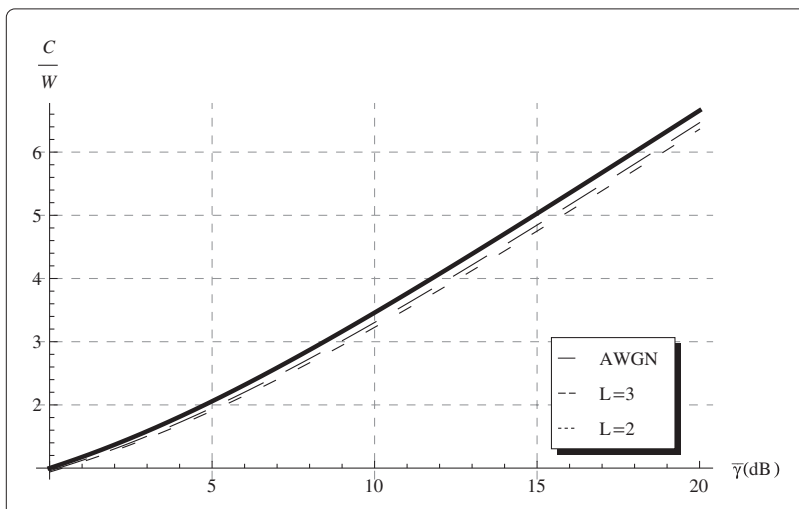

(a) Analytical results

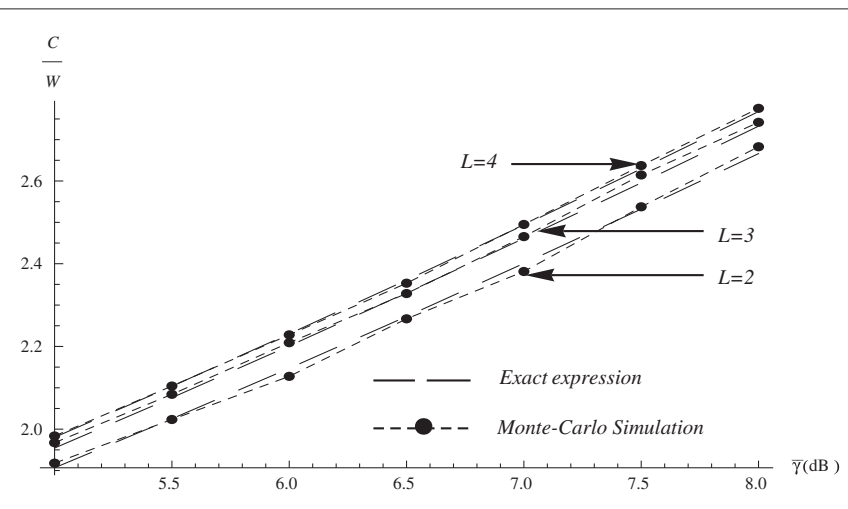

(b) Simulation and analytical results

Figure 4 Normalized average channel capacity versus average received SNR in a Rician L-path fading channel. (a) Analytical results. (b) Simulation and analytical results. 
Table 1 Average energies of the multipath fading (LOS and non LOS)

\begin{tabular}{cccc}
\hline $\boldsymbol{L}$ & $\mathbf{2}$ & $\mathbf{3}$ & $\mathbf{4}$ \\
\hline$s_{i}^{(2)}$ & $\frac{1}{4}, \frac{1}{4}$ & $\frac{1}{4}, \frac{1}{8}, \frac{1}{8}$ & $\frac{1}{4}, \frac{1}{8}, \frac{1}{16}, \frac{1}{16}$ \\
$2 \sigma^{2}$ & $\frac{1}{4}$ & $\frac{1}{6}$ & $\frac{1}{8}$ \\
$\lambda$ & 2 & $\sqrt{6}$ & $2 \sqrt{2}$ \\
$\beta$ & $2 \sqrt{2}$ & $2 \sqrt{3}$ & 4 \\
\hline
\end{tabular}

given in Table 1. By simulation, the series in expression (21) will converge after the 10th first terms for $L=2,3$ and after 30th first terms for $L=4$ which contribute to the computational complexity reduction.

Since the received power in the case of Rician fading is greater than that in the case of Rayleigh, so the required Rake receiver finger number to reach the AWGN capacity must be less or equal to 4 .

The Figure 5, shows the evolution of the PDF (17) as function as the number of resolvable paths $L$. This Figure has been plotted for the values given by the Table 1 . As $L$ is greater, the normalized amplitude of Rician multipath fading approaches to 1 . Furthermore, the Rake receiver may eliminate practically all fading and the couple channel plus Rake receiver has a behavior like that of a gaussian channel.

\section{Nakagami- $m$ multipath fading channel}

Let $N$ be the Nakagami- $m$ distributed RV of average energy $E\left[N^{2}\right]=2 \sigma^{2}$, and fading parameter $m=$ $\frac{\left(2 \sigma^{2}\right)^{2}}{E\left[\left(N^{2}-2 \sigma^{2}\right)^{2}\right]} \geq \frac{1}{2}$. The PDF of $N$ is given by [24]

$$
p(r)=\frac{2}{\Gamma(m)}\left(\frac{m}{2 \sigma^{2}}\right)^{m} r^{2 m-1} e^{-\frac{m r^{2}}{2 \sigma^{2}}}
$$

The square of a Nakagami distribution $\Omega=N^{2}$ is a gamma distribution $\Gamma(\alpha, \beta)$ of parameters $\alpha=m$ and $\beta=\frac{2 \sigma^{2}}{m}$ and PDF

$$
p(\gamma)=\frac{\left(\frac{m}{2 \sigma^{2}}\right)^{m}}{\Gamma(m)} \gamma^{m-1} e^{-\frac{m \gamma}{2 \sigma^{2}}}
$$

In the case of this fading, we show the expression of the channel capacity for two cases:

- The received average energies are equal ( $\sigma_{i}=\sigma$, $\forall i \leqslant L)$,

- The received average energies are not necessarily equal. This case generalizes the first one.

\section{Case 1: received average energies are equal}

Let $\left(\left|N_{l}\right|\right)_{1 \leq l \leq L}$ be RVs Nakagami- $m$ distributed of the same average energy $2 \sigma^{2}$. Since the distribution of the of two independent gamma RVs of parameters $\left(\alpha_{1}, \beta\right)$ and $\left(\alpha_{2}, \beta\right)$ is a gamma distribution of parameters $\left(\alpha_{1}+\right.$ $\left.\alpha_{2}, \beta\right)$ [25], the RV $\sum_{l=1}^{L}\left|N_{l}\right|^{2}$ is a gamma distribution of parameters $\left(m L, \frac{2 \sigma^{2}}{m}\right)$ and mean $2 L \sigma^{2}$.

Furthermore, $\frac{1}{\sqrt{2 L} \sigma} \sqrt{\sum_{l=1}^{L}\left|N_{l}\right|^{2}}$ is a normalized Nakagami$m$ distribution of fading parameter $m L$ and PDF

$$
f(r)=\frac{2}{\Gamma(m L)}(m L)^{m L} r^{2 m L-1} e^{-m L r^{2}}
$$

It follows the expression of the normalized capacity of the equivalent channel model given by the expression (3) is [6]:

$$
C=W \frac{\left(\frac{m L}{\bar{\gamma}}\right)^{m L}}{\ln (2) \Gamma(m L)} G_{2,3}^{3,1}\left[\begin{array}{l|l}
\frac{m L}{\bar{\gamma}} & \begin{array}{l}
-m L, 1-m L \\
0,-m L,-m L
\end{array}
\end{array}\right]
$$

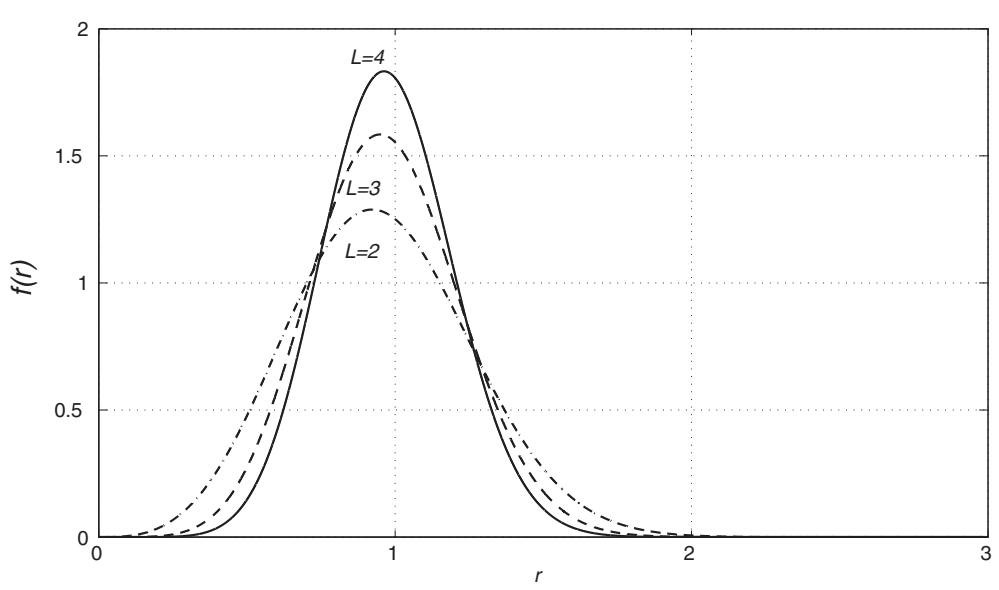

Figure 5 PDF of the Normalized fading amplitude in a Rician 4-path fading channel. 
This expression generalizes again that of Sagias et al. and that given by (13). Indeed, for $m=1,(25)$ reduces to the average capacity of the Rayleigh fading equivalent channel given by (13) and for $L=1$ we got the channel capacity of the Nakagami fading channel (one path) shown in [6]. In order to justify the limit of three fingers in Rake receiver, we present in both Figure 6a,b, for a given value of $m$, the normalized capacities of Nakagami- $m$ multipath fading channel obtained analytically from (25).

It's clear that for $m \geq 1$ (Figures $2 \mathrm{a}$ and $6 \mathrm{a}, \mathrm{b}$ ), $L=4$ appears to be sufficient to approach the AWGN channel.

In other ways, Figure 7a shows the effect of Nakagami fading parameter $m$ on the maximal spectral efficiency for the given value of $L$. The greater is $m$, the better is the spectral efficiency and approaches to the AWGN one. The Figure $7 \mathrm{~b}$ depict the average capacity computed by Monte-Carlo simulation generating 2500 Nakagami$m$ distributed random values to validate the analytical expression as presented above in (25).

Practically, for 3-path channel and $m=3$, the fading channel behaves like an AWGN channel.

In Figure 8, the PDF of the normalized fading amplitude given by (24) in the case of $m=1.5$ and both $L=4$ and $L=3$, is plotted as a function as fading amplitude. The multipath fading in this case is equivalent of Nakagami fading channel (one path) with parameter $m L$ (6 and 4.5, respectively). As $m L$ increases, the fading amplitude approaches to 1 and then the channel is approximatively equivalent to the AWGN one.

\section{Case 2: received average energies are not equal}

Let $\left(N_{i}\right)_{i \leqslant L}$ be $L$ independent Nakagami- $m$ RVs of average energies $2 \sigma_{i}^{2}$, and the same fading parameter $m$. The square of the RV $N_{i}$ is a gamma distribution $\Gamma\left(m, \beta_{i}=\frac{2 \sigma_{i}^{2}}{m}\right)$. Furthermore, the PDF of the sum $\sum_{i=1}^{L} N_{i}^{2}$ of gamma RVs is given by [25]

$$
p(r)=\frac{r^{L m-1}}{\prod_{i=1}^{L} \beta_{i}^{m}} e^{-\frac{r}{\beta}} \sum_{k=0}^{+\infty} \varepsilon_{k} r^{k}, \quad r \geqslant 0,
$$

where $\beta=\frac{2 \sigma^{2}}{m}=\min _{1 \leqslant i \leqslant L} \beta_{i}, \quad \varepsilon_{k}=\frac{\delta_{k}}{\beta^{k} \Gamma(L m+k)}$ and $\delta_{k}$ is computed recursively by this formulas:

$$
\delta_{k}=\left\{\begin{array}{c}
1, \text { si } k=0 \\
\frac{m}{k} \sum_{i=1}^{k} \delta_{k-i} \sum_{j=1}^{L}\left(1-\left(\frac{\sigma}{\sigma_{j}}\right)^{2}\right)^{i}
\end{array}\right.
$$

Thus, the PDF of the normalized fading amplitude $Z=$ $\frac{1}{\xi} \sqrt{\sum_{i=1}^{L} N_{i}^{2}}$, where $\xi=\sqrt{2 \sum_{i=1}^{L} \sigma_{i}^{2}}$ is given by:

$$
p(z)=\frac{2 \xi^{2 L m}}{\prod_{i=1}^{L} \beta_{i}^{m}} z^{2 L m-1} e^{-\frac{z^{2} \xi^{2}}{\beta}} \sum_{k=0}^{+\infty} \xi^{2 k} \varepsilon_{k} z^{2 k}
$$

The Shannon capacity of the Nakagami- $m$ multipath fading channel in the case of unequal average energies is then:

$$
C=W \frac{\xi^{2 L m}}{\prod_{i=1}^{L} \beta_{i}^{m}} \sum_{k=0}^{+\infty} C_{k}
$$

where $C_{k}=\frac{\xi^{2 k} \varepsilon_{k}}{\bar{\gamma}^{k+L m}} \int_{0}^{+\infty} \log _{2}(1+\gamma) \gamma^{k+L m-1} e^{-\frac{\gamma \xi^{2}}{\beta \bar{\gamma}}} d \gamma$. Using the transformation formulas (10), (11), and (12), we obtain:

$$
C_{k}=\frac{\xi^{2 k} \varepsilon_{k}}{\bar{\gamma}^{k+L m} \ln (2)} G_{2,3}^{3,1}\left[\begin{array}{l|l}
\frac{\xi^{2}}{\beta \bar{\gamma}} & \begin{array}{l}
-k-m L, 1-k-m L \\
0,-k-m L,-k-m L
\end{array}
\end{array}\right]
$$

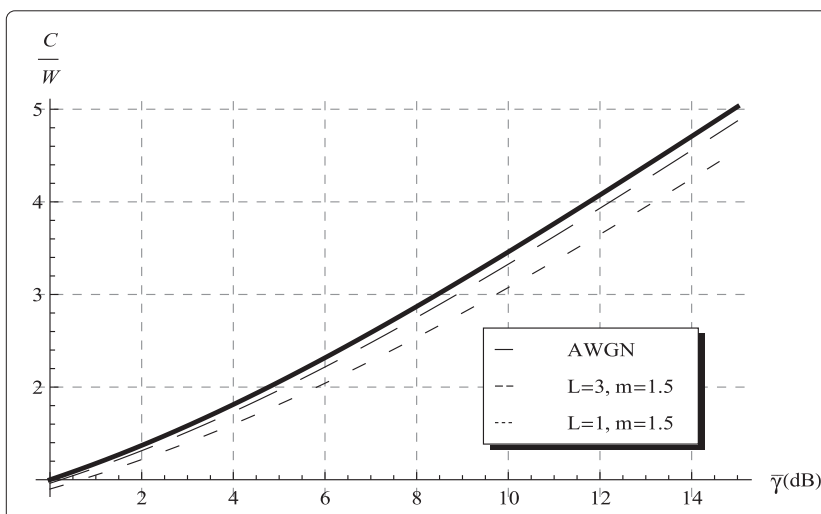

(a) $\mathrm{m}=1.5$

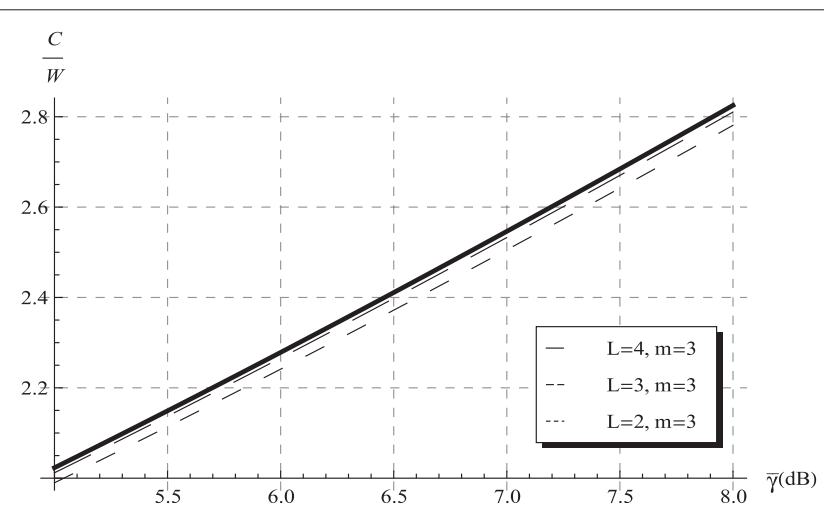

(b) $m=3$

Figure 6 Normalized average channel capacity versus average received SNR in Nakagami- $m$ L-path fading channel. (a) $m=1.5$. (b) $m=3$. 


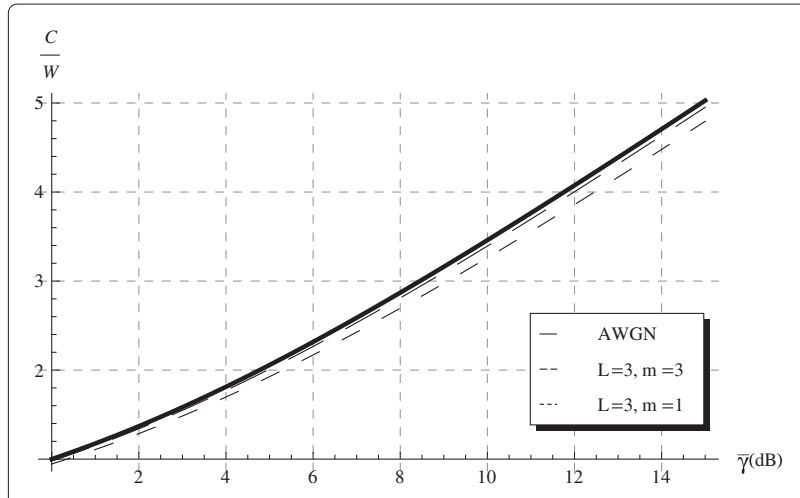

(a) Analytical expression

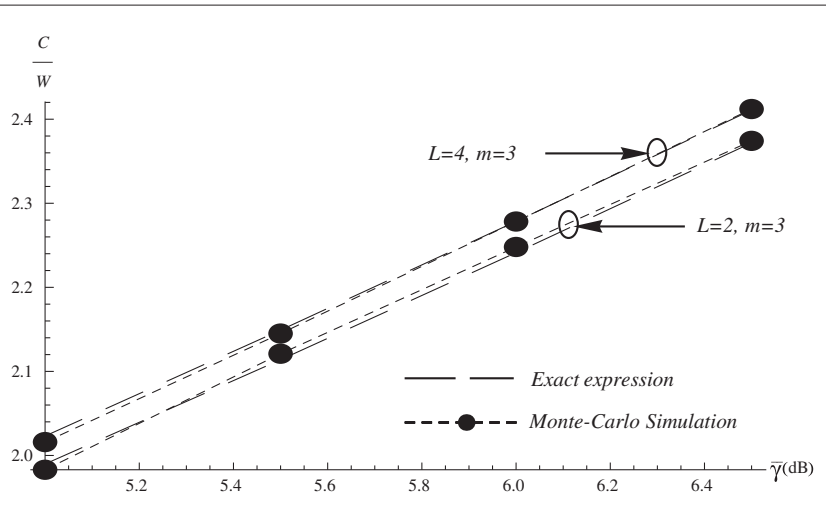

(b) Simulation and analytical expressions

Figure 7 Normalized average channel capacity versus average received SNR in Nakagami- $m$ L-path fading channel. (a) Analytical expression. (b) Simulation and analytical expressions.

Thus:

$$
\begin{aligned}
C= & \frac{W \phi^{L m}}{\ln (2)} \prod_{i=1}^{L}\left(\frac{\sigma}{\sigma_{i}}\right)^{2 m} \sum_{k=0}^{+\infty} \frac{\phi^{k} \delta_{k}}{\Gamma(L m+k)} \\
& \times G_{2,3}^{3,1}\left[\begin{array}{l|l}
\phi & \begin{array}{l}
-k-m L, 1-k-m L \\
0,-k-m L,-k-m L
\end{array}
\end{array}\right]
\end{aligned}
$$

where:

$$
\phi=\frac{\xi^{2}}{\beta \cdot \bar{\gamma}}=\frac{m \sum_{i=1}^{L} \sigma_{i}^{2}}{\bar{\gamma} \sigma^{2}}
$$

This expression generalizes (25). Indeed, in the case of the same average energies $\left(\sigma_{i}=\sigma\right), \phi=\frac{m L}{\bar{\gamma}}$ and $\delta_{k}=0$ $(k>0)$.

The Figure 9a compares the normalized Shannon capacity, computed analytically from (31), for the Nakagami-3 3-path fading channel and average energies $2 \sigma_{i}^{2}=\frac{1}{4}, \frac{1}{4}, \frac{1}{2}$. The capacity will converge after the $K=15$ th first terms of the infinite series presented above in (31), and is upper bounded by AWGN capacity. Figure 9b shows the convergence of capacity based on infinite series (31) to the simulated one computed from (5).

The Figure 10 depicts the PDFs of the normalized 3-path fading amplitude in both constants and various average energies $\left(2 \sigma_{i}^{2}=\frac{1}{4}, \frac{1}{4}, \frac{1}{2}\right)$. The curves have been plotted for $m=3$, respectively, from (24) and (28). By simulation, the PDF (28) will converge after the 15th first terms of the series. Furthermore, in the two cases, the fading amplitude of the multipath channel approaches to 1 .

\section{Weibull multipath fading channel}

Weibull fading, based on the Weibull distribution, is a simple statistical model of fading both in indoor and outdoor wireless communications.

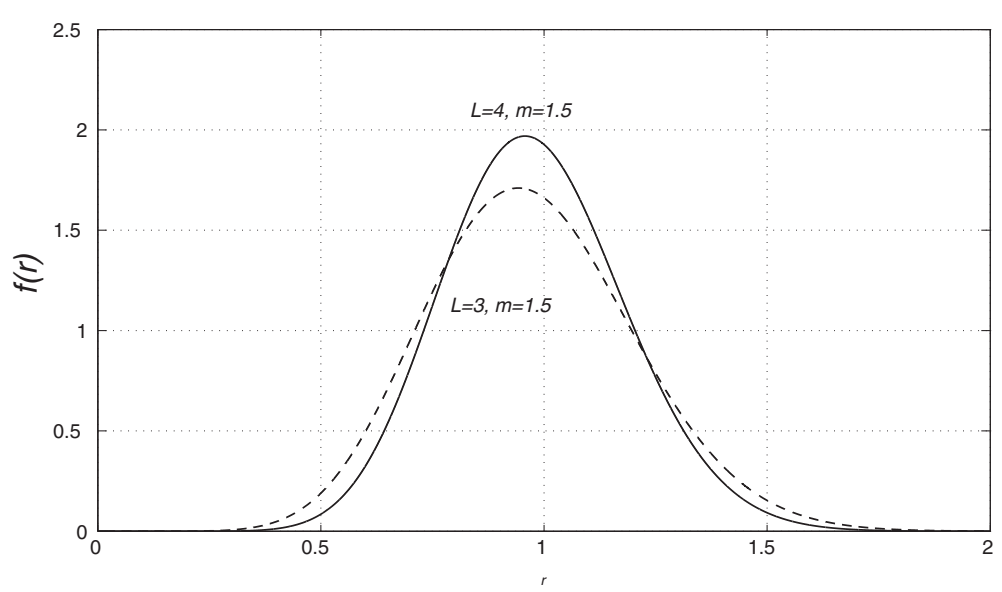

Figure 8 PDFs of the normalized fading amplitude in Nakagami- $m L$-path fading channel $(m=1.5)$. 


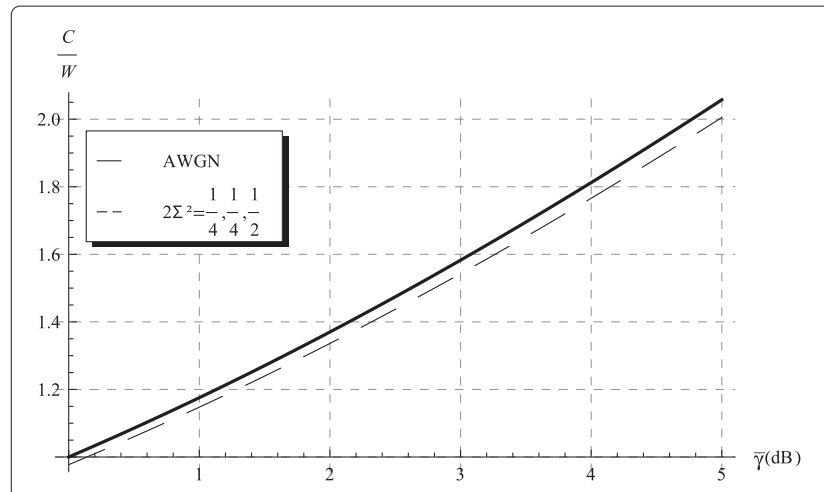

(a) Analytical expression $\left(2 \sigma_{i}^{2}=\frac{1}{4}, \frac{1}{4}, \frac{1}{2}\right)$

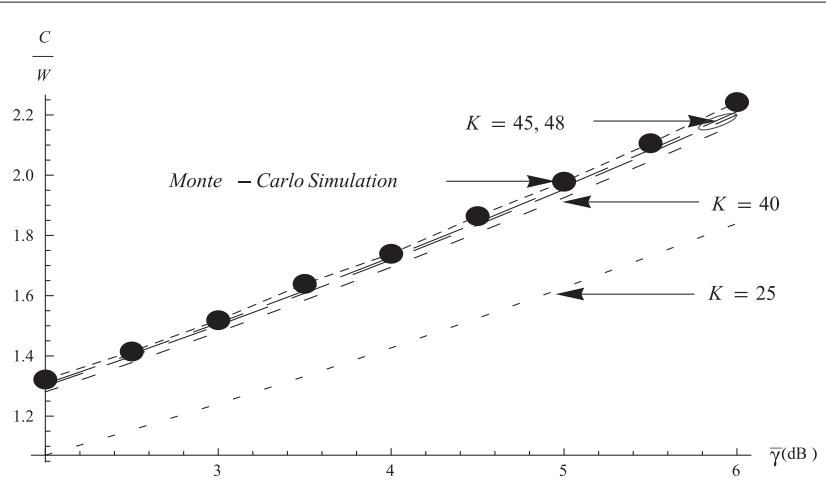

(b) Analytical and simulation expressions $\left(2 \sigma_{i}^{2}=\frac{1}{8}, \frac{1}{8}, \frac{3}{4}\right)$

Figure 9 The Shannon capacity per bandwidth of the Nakagami-3 multipath fading channel $(\boldsymbol{L}=\mathbf{3})$. (a) Analytical expression $\left(2 \sigma_{i}^{2}=\frac{1}{4}, \frac{1}{4}, \frac{1}{2}\right)$. (b) Analytical and simulation expressions $\left(2 \sigma_{i}^{2}=\frac{1}{8}, \frac{1}{8}, \frac{3}{4}\right)$.

A Weibull distribution $W_{\beta}$ with shape parameter $\beta$ can be transformed to a Rayleigh distribution $R$ and vice versa:

$$
W_{\beta}=R^{\frac{2}{\beta}}
$$

Let $\lambda$ be the scale parameter of $W_{\beta}$. The PDF of the Weibull distributed RV of parameter $\beta$ with mean $E[R]=$ $\lambda \Gamma\left(1+\frac{1}{\beta}\right)$ and average energy $E\left[R^{2}\right]=\lambda^{2} \Gamma\left(1+\frac{2}{\beta}\right)$ is given by:

$$
p(\omega)=\frac{\beta}{\lambda}\left(\frac{\omega}{\lambda}\right)^{\beta-1} e^{-\left(\frac{\omega}{\lambda}\right)^{\beta}}, \quad \omega \geqslant 0
$$

Furthermore, the square of a Weibull distribution $\Omega=$ $W^{2}$ is a Weibull distribution of shape parameter $\frac{\beta}{2}$ and mean $E[\Omega]=\lambda^{2} \Gamma\left(1+\frac{2}{\beta}\right)$. Thus, the normalized $A_{L}^{2}$ distribution in the case of Weibull $L$-path fading channel is a sum of $L$ i.i.d Weibull distribution of the same parameters. Its PDF is generally unknown excepted some easy cases [26]. In [27], it has been shown that the sum of Weibull RV can be closely approximated by the $\alpha-\mu$ distribution where $\left(\alpha=\frac{\beta}{2}\right.$ and $\left.\mu=L\right)$ where the PDF is given by:

$$
p(\omega)=\frac{\beta}{2 \lambda \Gamma(L)}\left(\frac{\omega}{\lambda}\right)^{\frac{L \beta}{2}-1} e^{-\left(\frac{\omega}{\lambda}\right)^{\frac{\beta}{2}}}, \quad \omega \geqslant 0,
$$

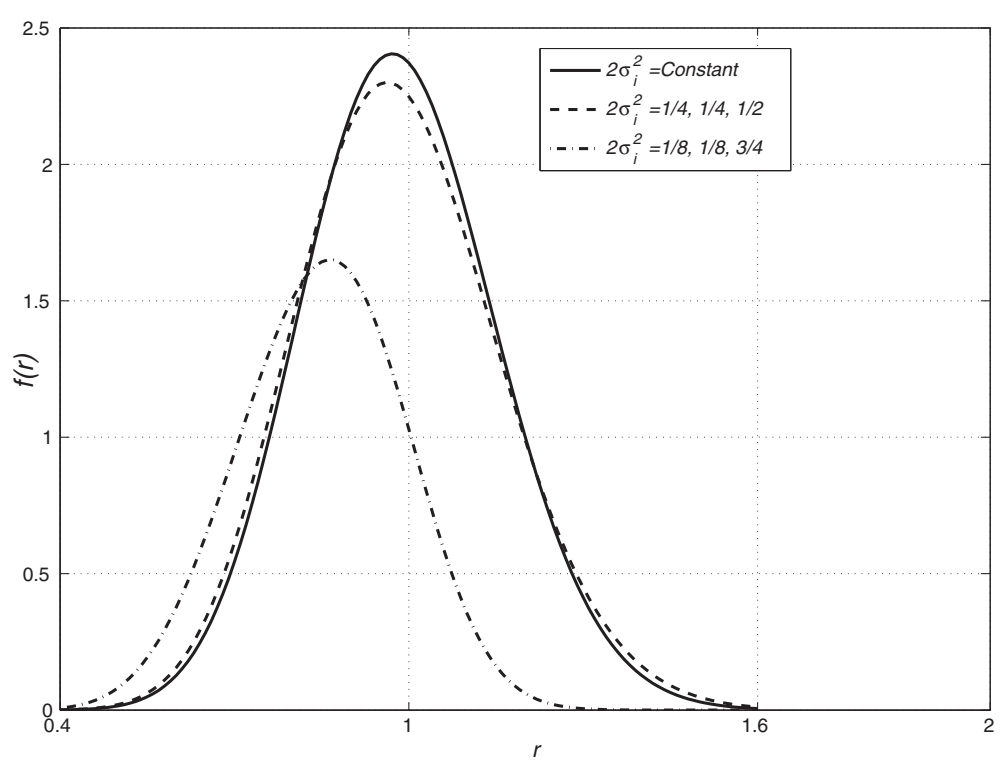

Figure 10 PDF of the Normalized fading amplitude in Nakagami 3-path fading channel. 
where

$$
E\left[A_{L}^{2}\right]=\lambda \frac{\Gamma\left(L+\frac{2}{\beta}\right)}{\Gamma(L)}
$$

Furthermore:

$$
\lambda=\frac{\Gamma(L)}{\Gamma\left(L+\frac{2}{\beta}\right)}
$$

The PDF of the approximated normalized fading $A_{L}$ is then:

$$
p(\omega)=\frac{\beta}{\sqrt{\lambda} \Gamma(L)}\left(\frac{\omega}{\sqrt{\lambda}}\right)^{L \beta-1} e^{-\left(\frac{\omega}{\sqrt{\lambda}}\right)^{\beta}}, \quad \omega \geqslant 0
$$

By replacing (38) into (5) and using the same change of variable $\gamma=\bar{\gamma} \omega^{2}$, the approximated average Shannon capacity for the Weibull $L$-path fading channel can be approximated by:

$$
\begin{aligned}
C= & W \frac{\beta}{2 \ln (2) \Gamma(L) \lambda \bar{\gamma}} \int_{0}^{+\infty} e^{-\left(\frac{\gamma}{\lambda \cdot \bar{\gamma}}\right)^{\frac{\beta}{2}}} \\
& \times G_{2,2}^{1,2}\left[\begin{array}{l|l}
\gamma & 1,1 \\
1,0
\end{array}\right] G_{0,1}^{1,0}\left[\left(\frac{\gamma}{\lambda \bar{\gamma}}\right)^{\frac{\beta}{2}} \mid 0\right] d \gamma
\end{aligned}
$$

This integral can be solved in closed-form ([23], /HypergeometricFunctions/MeijerG/21/02/03/01/), [28]:

$$
\begin{aligned}
C= & W \frac{\beta(\lambda \bar{\gamma})^{-\frac{L \beta}{2}} \sqrt{k}}{2 \Gamma(L) \ln (2) l(2 \pi)^{l+\frac{k}{2}-\frac{3}{2}}} \\
& \times G_{2 l, k+2 l}^{k+2 l, l}\left[\left(k(\lambda \bar{\gamma})^{\frac{\beta}{2}}\right)^{-k} \mid \Psi\left(k,-\frac{L \beta}{2}, l\right)\right],
\end{aligned}
$$

Table 2 The parameters of Figure 11

\begin{tabular}{cccc}
\hline$\beta$ & 1.5 & 2 & 2.8 \\
$l$ & 3 & 1 & 7 \\
$k$ & 4 & 1 & 5 \\
\hline
\end{tabular}

where

$$
\Psi(k, p, l)=\left[\begin{array}{c}
\Phi(p, l), \Phi(1+p, l) \\
\Phi(0, k), \Phi(p, l), \Phi(p, l)
\end{array}\right]
$$

and $\Phi(p, l)=\frac{p}{l}, \frac{p+1}{l}, \ldots, \frac{p+l-1}{l}$ and $l, k$ are the two small positive integers co-prime such that $\frac{l}{k}=\frac{\beta}{2}$.

This formulas generalizes the capacity obtained for Rayleigh multipath fading channel (13) by setting $\beta=$ $2\left(k=l=1, \lambda=\frac{1}{L}\right)$, and the Shannon capacity of Weibull fading channel (one path) given by Sagias et al. for $L=1$ [6].

The Figure 11a shows the effect of the Weibull fading parameter $\beta$ on the Shannon capacity of Weibull 4-path fading channel, plotted for the values of $l$ and $k$ given by the Table 2 from the expression (40).

As the number $\beta$ increases, as the fading disappears (expression (33)), and the capacity (40) becomes closer to that of the AWGN for four Rake receiver fingers and $\beta \geqslant 2.8$.

The Figure 11b depict the comparison between the analytical and simulation expressions by generating 2000 Weibull-distributed random numbers. It is shown that the approximated Shannon capacity given in (40) is very closed to the exact one.

The Figure 12 shows the approximated PDFs of the normalized 4-path fading amplitude for the values of $\beta$ given

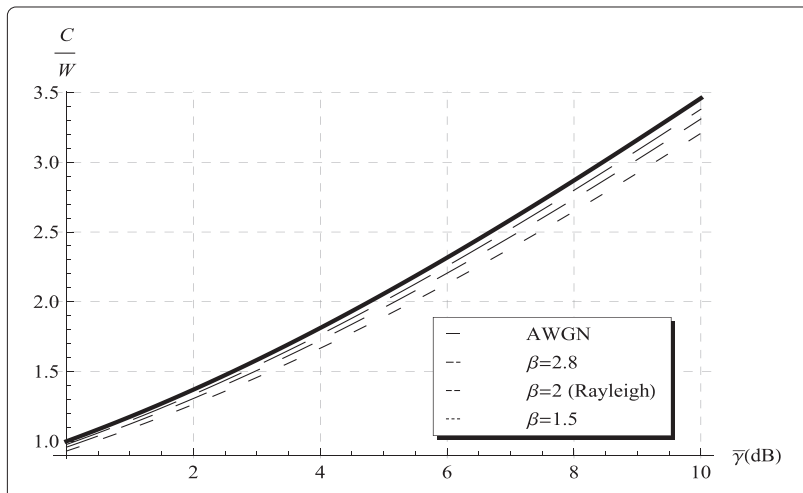

(a) Analytical expression $L=4$

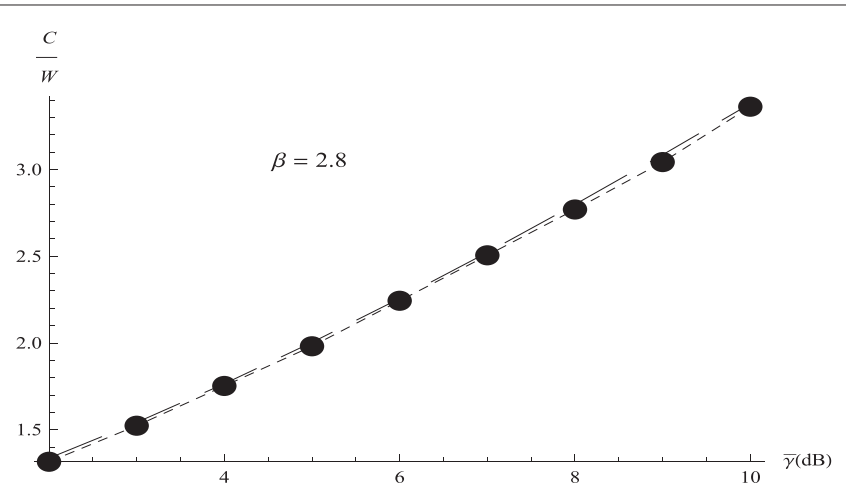

(b) Analytical and simulation expressions $(L=4)$

Figure 11 Normalized Shannon capacity of the Weibull- $\beta$ multipath fading channel. (a) Analytical expression $L=4$. (b) Analytical and simulation expressions $(L=4)$. 


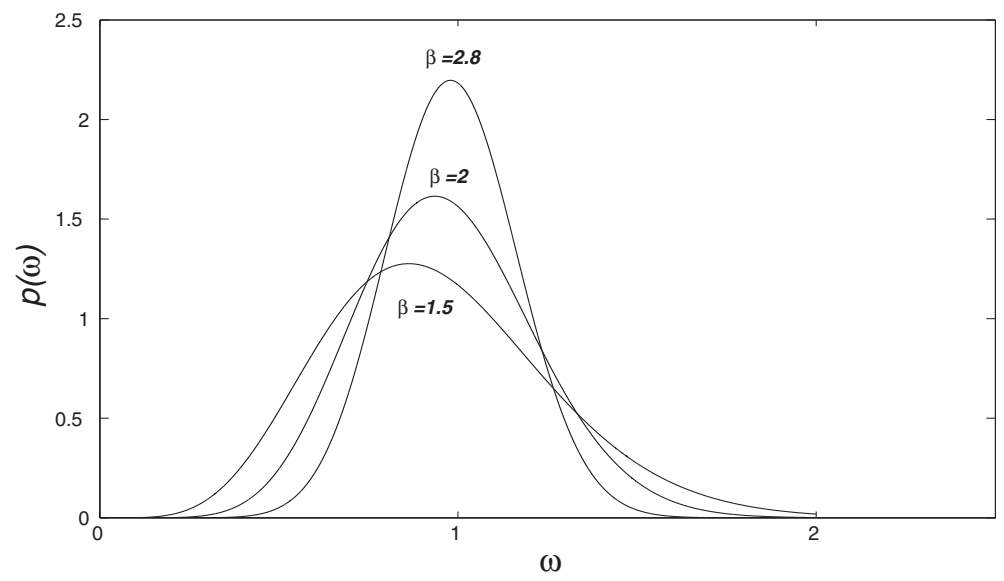

Figure 12 PDF of the approximated fading amplitude in Weibull 4-path fading channel.

by the Table 2 . The curves have been plotted from (38) and show that the multipath fading amplitude is close to 1 for large values of the shape parameter.

\section{Conclusion}

In this article, we have derived the closed-form expression of the channel capacity over Nakagami-m, Rician and Rayleigh multipath flat fading channel with $L$-branch $M R C$ receiver and an tight approximation for Weibull case in terms of Meijer G-functions. The DS-CDMA cellular system case was also considered. The results were validated using Monte-Carlo Simulations for all multipath fading channels studied. The plotted curves show that as $L$ increases, the capacity approaches the AWGN channel capacity. Furthermore, four branches are sufficient, in all cases, to eliminate the fading effect and then to achieve the maximum capacity.

\section{Appendix 1}

It is convenient to recall notations and definitions concerning Meijer G-function (see [21,23,28-30]). Let $a_{1}, \ldots, a_{n}, \ldots, a_{p}$ and $b_{1}, \ldots, b_{m}, \ldots, b_{q}$ be complex numbers which we designate by $\left(a_{p}\right)$ and $\left(b_{q}\right)$, respectively, for $0 \leq m \leq q, 0 \leq n \leq p$. The G-function of a complex variable $z$ is given by a Mellin-Barnes type integral:

$$
\begin{aligned}
& G_{p, q}^{m, n}(z)=G\left[z \mid \begin{array}{l}
z \\
a_{1}, \ldots, a_{n}, \ldots, a_{p} \\
b_{1}, \ldots, b_{m}, \ldots, b_{q}
\end{array}\right] \\
& =G\left[\begin{array}{l|l}
z & \left(a_{p}\right) \\
\left(b_{q}\right)
\end{array}\right]=\frac{1}{2 \pi i} \int_{L} g(s) z^{-s} d s,
\end{aligned}
$$

where

$$
g(s)=\frac{\prod_{j=1}^{m} \Gamma\left(b_{j}+s\right) \prod_{j=1}^{n} \Gamma\left(1-a_{j}-s\right)}{\prod_{j=n+1}^{p} \Gamma\left(a_{j}+s\right) \prod_{j=1+m}^{q} \Gamma\left(1-b_{j}-s\right)}
$$

We require the conditions that $a_{j}-b_{k}$ is not zero or a positive integer for $j=1, \ldots, n, k=1, \ldots, m$. In the integrand, an empty product is interpreted as unity. The path of integration $L$ if one of the following:

(i) Left loop beginning at $-\infty+\lambda i$ and ending at $-\infty+\delta i$ for $\lambda<\delta$ encircling once all the poles $-b_{j}-l$ (for $j=1 \ldots, m, l=0,1, \ldots$ ) leaving the poles $1-a_{j}+l$ (for $\left.j=1 \ldots, n, l=0,1, \ldots\right)$ to the right.

(ii) Right loop beginning at $\infty+\lambda i$ and ending at $\infty+\delta i$ for $\lambda>\delta$ encircling once all the poles $1-a_{j}+l$ (for $j=1 \ldots, m, l=0,1, \ldots$ ) leaving the poles $b_{j}-l$ (for $j=1 \ldots, n, l=0,1, \ldots)$ to the left.

(iii) A line with indentation beginning at $\lambda-i \infty$ ending at $\lambda+i \infty$ (for some real $\lambda$ ) separating the poles of the integrand $g$ like the cases (i) and (ii). We refer to $[29,30]$ for the existence of $G(z)$.

- For any $x$ ([23], /07.34.03.0228.01),

$$
e^{-x}=G_{0,1}^{1,0}[x \mid 0]
$$

- For any $x$ ([23], /07.34.03.0456.01),

$$
\ln (1+x)=G_{2,2}^{1,2}\left[\begin{array}{l|l}
x & 1,1 \\
1,0
\end{array}\right]
$$

- For $-\pi<\arg x \leq 0$ the modified Bessel function of the first kind ([23], /07.34.03.0230.01),

$$
I_{n}(x)=i^{-n} G_{0,2}^{1,0}\left[-\left.\frac{x^{2}}{4}\right|_{n / 2,-n / 2}\right]
$$

- The Mellin transform of a G-function is ([23], /07.34.21.0009.01),

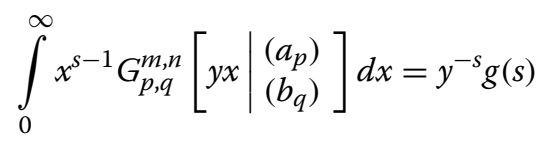


- The Mellin transform of a product of two G-functions is ([23], /07.34.21.0011.01), [28],

$$
\begin{aligned}
& \int_{0}^{\infty} x^{\alpha-1} G_{u, v}^{s, t}\left[\sigma x \mid \begin{array}{c}
\left(c_{u}\right) \\
\left(d_{v}\right)
\end{array}\right] G_{p, q}^{m, n}\left[\omega x \mid \begin{array}{c}
\left(a_{p}\right) \\
\left(b_{q}\right)
\end{array}\right] d x \\
& \quad=\sigma^{-\alpha} G_{p+v, q+u}^{m+t, n+s}\left[\frac{\omega}{\sigma} \mid \begin{array}{l}
\left(a_{n}\right), 1-\alpha-d_{1}, \ldots, 1-\alpha-d_{v}, a_{n+1}, \ldots, a_{p} \\
\left(b_{m}\right), 1-\alpha-c_{1}, \ldots, 1-\alpha-c_{v}, b_{m+1}, \ldots, b_{q}
\end{array}\right]
\end{aligned}
$$

\section{Competing interests}

The authors declare that they have no competing interests.

\section{Author details}

${ }^{1}$ ENSIAS, Mohammed V-Souissi University, Rabat, Morocco. ${ }^{2}$ ENSAM, Meknès, Morocco.

Received: 17 December 2011 Accepted: 12 September 2012

Published: 7 November 2012

\section{References}

1. CE Shannon, A mathematical theory of communication. Bell Syst. Tech. J. 27, 379-423; 623-656 (1948)

2. RG Gallager, Information Theory and Reliable Communication (John Wiley and Sons, Inc., New York, 1968)

3. MK Simon, M-S Alouini, Digital Communication Over Fading Channels, 2nd edn (Wiley, New York, 2005)

4. MS Alouini, A Goldsmith, Capacity of Rayleigh fading channels under different adaptive transmission and diversity-combining techniques. IEEE Trans. Veh. Technol.

48, 1165-1181 (1999)

5. RK Mallik, MZ Win, JW Shao, M-S Alouini, AJ Goldsmith, Channel capacity of adaptive transmission with maximal ratio combining in correlated Rayleigh fading. IEEE Trans. Wirel. Commun. 3(4), 1124-1133 (2004)

6. NC Sagias, GS Tombras, GK Karagiannidis, New results for the Shannon channel capacity in generalized fading channels. IEEE Commun. Lett. $\mathbf{9}(2)$, 97-99 (2005)

7. NC Sagias, GK Karagiannidis, DA Zogas, PT Mathiopoulos, GS Tombras, Performance analysis of dual selection diversity in correlated Weibull fading channels. IEEE Trans. Commun. 52, 1063-1067 (2004)

8. M Abramowitz, IA Stegun, Handbook of Mathematical functions, 55 (National Bureau of Standards - U.S. Government Printing Office, 1972)

9. GK Karagiannidis, NC Sagias, TA Tsiftsis, Closed-form statistics for the sum of squared Nakagami-m variates and its applications. IEEE Trans. Commun. 54(8), 1353-1359 (2004)

10. IS Ansari, F Yilmaz, M-S Alouini, O Kucur, in IEEE Int. Workshop on Sig. Proc Advan. in Wireless Comm. accepted in SPAWC'12, New results on the sum of gamma random variates with application to the performance of wireless communication systems over Nakagami-m fading channels available in http://arxiv.org/pdf/1202.2576.pdf

11. NC Sagias, FI Lazarakis, AA Alexandridis, KP Dangakis, GS Tombras, Higher order capacity statistics of diversity receivers. Wirel. Personal Commun. 56(4), 649-668 (2011)

12. S Khatalin, JP Fonseka, Capacity of correlated Nakagami-m fading channels with diversity combining techniques. IEEE Trans. Veh. Commun. 55(1), 142-150 (2006)

13. M Di Renzo, F Graziosi, F Santucci, Channel capacity over generalized fading channels: a novel MGF-based approach for performance analysis and design of wireless communication systems. IEEE Trans. Veh. Technol. 59(1), 127-149 (2010)

14. F Yilmaz, M-S Alouini, A unified MGF-based capacity analysis of diversity combiners over generalized fading channels. IEEE Trans. Commun. 60(3), 862-875 (2012). Available at http://arxiv.org/abs/1012.2596

15. NT Hai, SB Yakubovich, The Double Mellin-Barnes Type Integrals and their Applications to Convolution Theory (World Scientific, Singapore, 1992)

16. IS Ansari, S Al-Ahmadi, F Yilmaz, M-S Alouini, H Yanikomeroglu, A new formula for the BER of binary modulations with dual-branch selection over generalized-k composite fading channels. IEEE Trans. Comm. 59, 1291-1303 (2011)

17. M Xia, C Xing, Y Chung, S Aissa, Exact performance analysis of dual-hop semi-blind AF relaying over arbitrary Nakagami- $m$ fading channels. IEEE Trans. Wirel. Commun. 10(10), 3449-3459 (2011)

18. RD Cideciyan, E Eleftheriou, M Rupf, Concatenated Reed-Solomon/convolutional coding for data transmission in CDMA-Based cellular systems. IEEE Trans. Commun. 45, 1291-1303 (1997)

19. GL Stuber, Principles of Mobile Communications (Kluwer Academic Publishers, Mass, 1996)

20. JG Proakis, Digital Communications, 3rd edn. (McGraw-Hill, New York, 1995)

21. AP Prudnikov, YuA Brychkov, OI Marichev, Integrals and Series, Volume 3: More special functions (Gordon and Breach Science Publishers, New York, 1990)

22. NL Johnson, S Kotz, N Balakrishnan, Continuous Univariate Distributions, 2nd edn., vol. 2 (John Wiley \& Sons, Inc., New York, 1995)

23. Wolfram, The Wolfram functions site. Internet (online), http://functions. wolfram.com

24. M Nakagami, in Statistical Methods in Radio Wave Propagation, ed. by WC Hoffman. The m-Distribution, a general formula of intensity of rapid fading (New York:Pergamon Press, London, 1960), pp. 3-36

25. PG Moschopoulos, The distribution of the sum of independent Gamma random variables. Annals Inst. Stat. Math. Part A. 37, 541-544 (1985)

26. H Rinne, The Weibull distribution (CRC Press, Boca Raton, 2009)

27. JCS Santos Filho, MD Yacoub, Simple precise approximations to Weibull sums. IEEE Commun. Lett. 10(8), 614-616 (2006)

28. VS Adamchik, OI Marichev, in Proc. Int. Conf. on Symbolic and Algebraic Computation, The algorithm for calculating integrals of hypergeometric type functions and its realization in REDUCE system (Tokyo, Japan, 1990), pp. 212-224

29. AM Mathai, RK Saxena, Generalized hypergeometric functions with applications in statistics and physical sciences. Notes of Mathematics Series No. 348, Heidelberg, Germany, (1973)

30. A Erdélyi, W Magnus, F Oberhettinger, FG Tricomi, Higher Transcendental Functions, vol. I. (McGraw-Hill, New York, 1953)

\section{doi:10.1186/1687-1499-2012-336}

Cite this article as: El Bouanani et al:: New results for Shannon capacity over generalized multipath fading channels with MRC diversity. EURASIP Journal on Wireless Communications and Networking 2012 2012:336. 